\title{
Supramolecular Compartmentalized Hydrogels via Polydopamine Particle-Stabilized Water-in-Water Emulsions
}

\author{
Jianrui Zhang, ${ }^{\dagger}$ Baris Kumru, ${ }^{\dagger}$ and Bernhard V. K. J. Schmidt*, ${ }^{\dagger}$ \$(1) \\ ${ }^{\dagger}$ Department of Colloid Chemistry, Max Planck Institute of Colloids and Interfaces, Am Mühlenberg 1, 14476 Potsdam, Germany \\ ${ }^{\ddagger}$ School of Chemistry, University of Glasgow, Glasgow G12 8QQ U.K.
}

\section{Supporting Information}

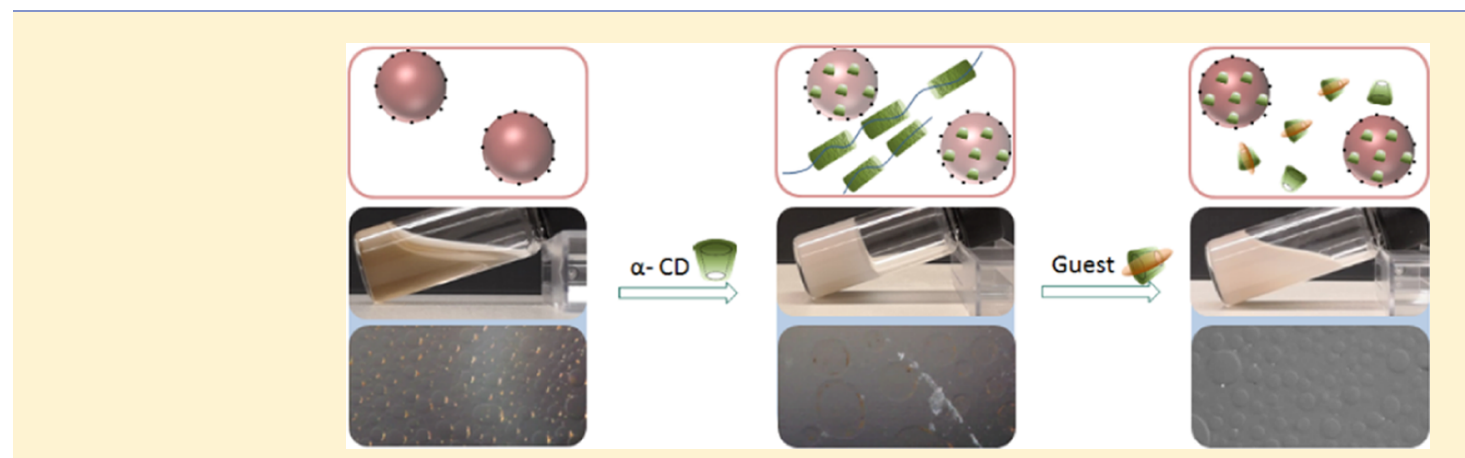

ABSTRACT: Compartmentalized hydrogels constitute a significant research area, for example, for catalytic and biomedical applications. As presented here, a generic method is used for compartmentalization of supramolecular hydrogels by using waterin-water emulsions based on aqueous two-phase systems. By forming the supramolecular hydrogel throughout the continuous phase of all-aqueous emulsions, distinct, microcompartmentalized materials were created. The basis for the presented compartmentalized water-in-water hydrogels is polydopamine particle-stabilized water-in-water emulsions from dextran and poly(ethylene glycol) (PEG). Addition of $\alpha$-cyclodextrin ( $\alpha$-CD) led to supramolecular complexation with PEG and subsequent hydrogel formation showing no signs of creaming. Due to the supramolecular nature of the compartmentalized hydrogels, selective network cleavage could be induced via competing guest addition, while keeping the emulsion substructure intact.

\section{INTRODUCTION}

Compartmentalization contributes an important role in a variety of biological and industrial processes. ${ }^{1}$ For instance, it allows organelles to perform synthetic tasks simultaneously inside eukaryotic cells by prohibiting mutual interference. ${ }^{2}$ Thus, the natural concept of compartmentalization has become a significant research topic in polymer and colloid science, e.g., in the endeavor for synthetic cells. ${ }^{3,4}$ Additionally, compartmentalization is crucial for encapsulation, drug delivery, and release of active flavor ingredients, as well as for structuring different materials through templating and scaffolding. ${ }^{5,6}$ For synthetic processes, compartmentalization is generally achieved either by emulsification of immiscible phases or through encapsulation of soft matter inside of vesicles. ${ }^{7-9}$ However, there are some limitations in the common approaches. For example, emulsification techniques are widely used for constituting hydrophobic domains in aqueous phases but are inherently limited in terms of the possible number of accessible types of compartments. ${ }^{10,11}$ On the other hand, vesicle-based approaches can effectively encapsulate various aqueous phases within their membranes and can be used to create numerous different coexisting microcompartments with excellent control over their composition. ${ }^{12}$ Nonetheless, while hydrophobic lipid- or polymer-based membranes grant vesicles their outstanding properties, they also seriously limit the transport of many polar solutes. Furthermore, in contrast to emulsions, which can be generated and stabilized relatively easily even on a large scale, preparation of loaded vesicles can be a long, not easily scalable process. Therefore, there is a need for a simple and elegant approach that allows the formation of microcompartments containing polar molecules without introducing any barriers.

One of the most promising directions for barrier-less structuring of aqueous media is to employ aqueous twophase systems (ATPS). ${ }^{13,14}$ ATPS are two-component waterbased mixtures generally containing two incompatible polymers and/or salts that can form distinct macroscopic aqueous phases. ${ }^{15,16}$ Aqueous two-phase systems are widely used in biotechnology for extraction and separation of biomolecules, organelles, and even living cells, as they offer a large number of mild, fully aqueous environments. ${ }^{17}$ Recently, Keating and co-workers exploited aqueous-phase separation as a tool for compartmentalizing biomolecules by encapsulating aqueous two- and three-phase systems within lipid vesicles to

Received: April 15, 2019

Revised: July 30, 2019

Published: August 2, 2019 
Scheme 1. Schematic Overview of Compartmentalized Hydrogel Formation

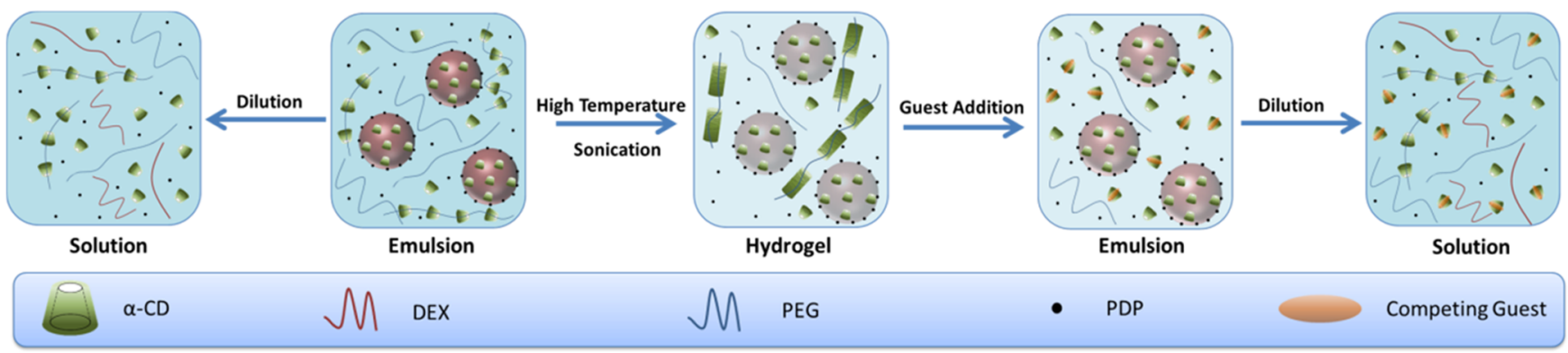

create prototype artificial cells. ${ }^{18-20}$ Several other examples of stabilization of water-in-water emulsions using various nanoparticles have also been reported. ${ }^{21-23}$ However, these methods have some limitations and cannot be specifically applied to form every stable arbitrary ATPS emulsion. An alternative surfactant-free route to stabilize emulsions has been recently demonstrated by the group of Ulijn, who have generated stable oil-in-water emulsions by creating an interfacial compartmentalized gel network around the dispersed droplets. $^{24,25}$ Therefore, formation of a supramolecular hydrogel network within ATPS emulsions should allow capturing their fine structure, resulting in microcompartmentalized, fully aqueous hydrogel materials. ${ }^{26}$ For example, van Esch and co-workers presented a completely water-based compartmentalized hydrogel formed from hydrazine hydrogelators. $^{27}$ In a similar way, Nicolai and co-workers recently showed water-in-water hydrogels stabilized by cellulose nanocrystals. ${ }^{28}$ Also, this structuring approach offers control over the composition of each compartment type by directing the partitioning of objects to be encapsulated, which is useful for the introduction of specific molecules, e.g., enzymes.

Certainly, it would be of practical interest if the same particles that stabilize the dispersed droplets against coalescence can also be used to produce compartmentalized hydrogels and thus stabilize against creaming or sedimentation. It was recently shown that this can be done successfully for w/ $\mathrm{w}$ emulsions formed by mixing aqueous solutions of dextran and poly(ethylene glycol) (PEG) in the presence of protein microgels. ${ }^{29}$ Excess microgels in the continuous phase could be induced to aggregate and form a network by adding salt or reducing the $\mathrm{pH}$, which reduces the electrostatic repulsion between the microgels. The $\mathrm{w} / \mathrm{w}$ emulsions formed by mixing aqueous solutions of pullulan and $\mathrm{PEG}^{21}$ or gelatin and dextran $^{23}$ in the presence of clay particles were found to be stable against the sedimentation of the dispersed droplets because aggregated clay particles attached to the droplet surface connected the droplets into a space-filling network. Compartmentalized hydrogels can be prepared via solid particle-stabilized $\mathrm{w} / \mathrm{w}$ emulsions, which is promising as an encapsulating structure as well as a scaffold for tissue engineering. ${ }^{30}$ Moreover, this kind of multicompartment structure offers specific spaces for encapsulation of cargo for drug delivery. ${ }^{31}$

Additionally, various kinds of polymer inclusion complexes (PICs) formed by noncovalent host-guest interactions have been extensively reported and investigated as useful building blocks for constructing supramolecular structures in hydrogel preparation. $^{32-35}$ Particularly, cyclodextrins (CDs) have been the most widely used host molecules. They have been the subjects of extensive investigation; one of the most notable features is that they form inclusion complexes with a wide variety of low-molecular-weight compounds ranging from nonpolar hydrocarbons to polar carboxylic acids and amines. So they are water-soluble and capable of selectively including a wide range of guest molecules. ${ }^{36,37}$ Harada and co-workers have introduced many PICs (or pseudo-polyrotaxanes) by a series of combinations between CDs, usually $\alpha$-, $\beta$-, and $\gamma$-CD, which consist of 6,7 , and 8 glucose units, respectively, and the corresponding linear polymers. ${ }^{32} \mathrm{Huh}$ and co-workers have also studied supramolecular-structured hydrogels on the basis of the inclusion complexation between PEG-grafted dextran and $\alpha$-CDs in aqueous media. ${ }^{38}$ They have fabricated polymer-polymer composites and blends with normally incompatible polymers using such a PIC formation. Our group recently showed the formation of a thermoadaptive hydrogel via $\alpha$-CD complexation of poly(vinylpyrrolidone)- $b$ poly(oligo ethylene glycol methacrylate). ${ }^{39}$ The gel formation of $\alpha$-CD and PEG is based on PIC formation and crystallization of the complexes via hydrogen bonding. As such, the crystalline domains act as crosslinking points in the hydrogel system. ${ }^{40}$

Polydopamine particles (PDP) are negatively charged particles produced by the alkaline hydrolysis of dopamine. The fabrication and broad applications of poly(dopamine) (PD) have rapidly advanced in recent years; PD displays many striking properties in polymer and material science, and, most importantly, it processes excellent biocompatibility. For instance, Cui et al. applied PD-coated capsules by emulsion templating to immobilize $\mathrm{pH}$-cleavable polymer-drug conjugates for intracellular drug delivery. ${ }^{41} \mathrm{PD}$ has impressive features for its surface modification. However, the exact structure of PD is still not clear yet. ${ }^{42}$ Notably, PDP can be used for emulsifying the aqueous two-phase system, specific for PEG and dextran. ${ }^{43}$ As shown before, PDP adsorb at the w/w phase boundary and effectively inhibit coalescence of dispersed aqueous emulsion droplets via the process of colloidal assembly at the interfaces. Particles can stabilize $\mathrm{w} / \mathrm{w}$ emulsions by forming a layer at the interface, which reduces the free energy. The stabilization of interfaces with particles is known as the Pickering effect and has been practically irreversible for $\mathrm{w} / \mathrm{w}$ interfaces. ${ }^{22,23}$

In the present contribution, we present a generic method for compartmentalizing aqueous media using aqueous-phase separation of incompatible polymers and the formation of a supramolecular hydrogel to obtain fixed compartments (Scheme 1). Therefore, PEG-dextran $\mathrm{w} / \mathrm{w}$ emulsions are formed in the presence of PDP and gelation via $\alpha$-CD is investigated. By forming the supramolecular hydrogel throughout the continuous phase of all-aqueous emulsions stabilized by PDP, distinctly, compartmentalized complexation between 

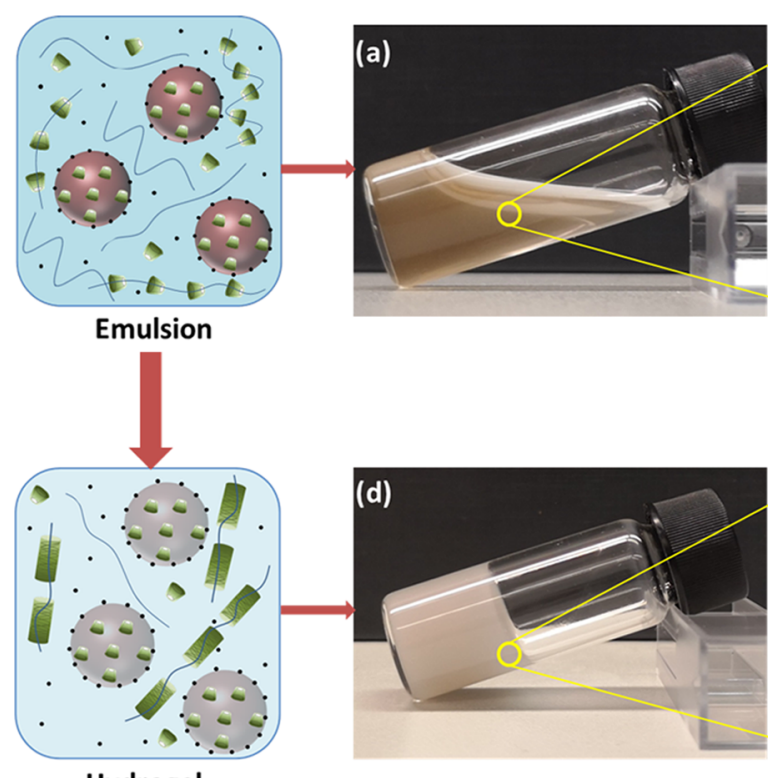

Hydrogel
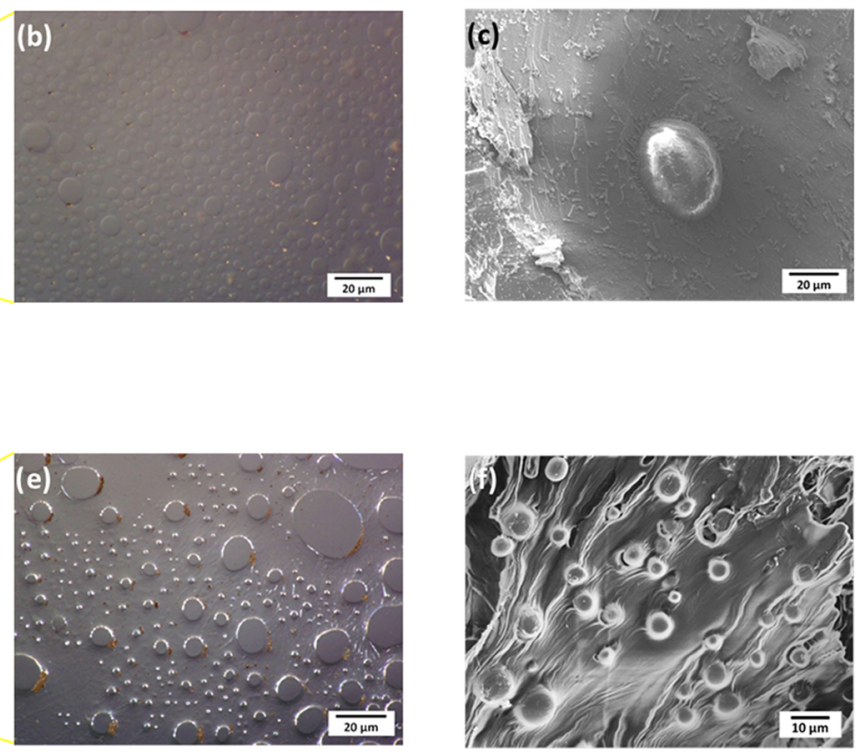

Figure 1. Images of the PDP $(0.2 \mathrm{~g} / \mathrm{L})$-stabilized $\mathrm{PEG}_{35 \mathrm{k}}(7 \mathrm{wt} \%) / \operatorname{dextran}_{40 \mathrm{k}}(3 \mathrm{wt} \%)$ water-in-water system $(140 \mathrm{mg} / \mathrm{mL}$ of $\alpha$-CD): Optical images of (a) a PDP-stabilized emulsion and (d) compartmentalized hydrogel after heating to $65{ }^{\circ} \mathrm{C}$ and cooling to ambient temperature, respectively; optical microscopy images of (b) emulsion droplets at ambient temperature and (e) emulsion droplets within a compartmentalized hydrogel; cryo-SEM image (c) of emulsion droplets; SEM image (f) of emulsion droplets within a compartmentalized hydrogel.

PEG and $\alpha$-CD is formed. Studies of the formed complexes are performed via X-ray diffraction (XRD), and the mechanical properties of the hydrogels are measured with oscillatory shear rheology. Moreover, we present the triggered disassembly of the hydrogels via competitive guest addition, while the emulsion stays intact keeping mobile compartmentalization of the solution. The emulsion can be demulsified in a further step leading to a complete loss of structuring. The hydrogels and emulsions are assessed via optical microscopy (OM) and confocal laser scanning microscopy (CLSM) showing a supramolecular compartmentalized hydrogel that can be selectively disassembled on various levels of structuring.

\section{EXPERIMENTAL SECTION}

Materials. Anthranilic acid (reagent grade, $\geq 98 \%$, Sigma Aldrich), $\alpha$-cyclodextrin $(\alpha$-CD; $\geq 98 \%$, Roth), dextran (40k, analytical grade; $100 \mathrm{k}$, analytical grade, all from Sigma Aldrich), dimethylsulfoxide (DMSO, extra dry, Acros Organics), dopamine hydrochloride (98\%, Sigma Aldrich), ethylenediamine resin (polymer-bound, 4.0-5.7 $\mathrm{mmol} / \mathrm{g}$, Sigma Aldrich), fluorescein isothiocyanate (FITC; 90\%, Sigma Aldrich), hydrochloric acid ( $\mathrm{HCl}$; fuming, Carl Roth), poly(ethylene glycol) (PEG; 20k, analytical grade; 35k, analytical grade; 40k, analytical grade, all from Sigma Aldrich), poly(ethylene glycol) diamine $\left(\mathrm{NH}_{2}-\mathrm{PEG}-\mathrm{NH}_{2} ; 2 \mathrm{k}\right.$, analytical grade, Sigma Aldrich) were obtained as indicated. Milli- $Q$ water was obtained from an Integra UV plus pure water system by SG Water (Germany). $\mathrm{PDP}^{42}$ and FITC-labeled $\mathrm{PEG}^{43}$ were obtained according to the literature (refer to Supporting Information).

Preparation of Compartmentalized Hydrogel. An example compartmentalized hydrogel was formed by mixing $\mathrm{PEG}_{35 \mathrm{k}}\left(c_{\mathrm{PEG}}=7\right.$ wt $\%, 0.90 \mathrm{~g})$ and dextran ${ }_{40 \mathrm{k}}\left(c_{\text {Dex }}=3 \mathrm{wt} \%, 0.40 \mathrm{~g}\right)$ with PDP- $\alpha$-CD suspension $(0.2 \mathrm{mg} / \mathrm{mL}$ of PDP, $140 \mathrm{mg} / \mathrm{mL}$ of $\alpha-\mathrm{CD}, 3 \mathrm{~mL})$. After mixing, the mixture was ultrasonicated for $2 \mathrm{~h}$ for dissolving and emulsification (Elmasonic S30H). Then, the sample was heated to 65 ${ }^{\circ} \mathrm{C}$ and cooled to ambient temperature for hydrogel formation. To study the temperature sensitivity, the procedure was evaluated further. After ultrasonication for $2 \mathrm{~h}$, the mixture was directly heated to $90{ }^{\circ} \mathrm{C}$ and then cooled to ambient temperature.
Stability of Emulsions and Compartmentalized Hydrogel. Dilution Study. Here, PEG $\left(c_{\mathrm{PEG}}=7 \mathrm{wt} \%, 0.90 \mathrm{~g}\right) /$ dextran $\left(c_{\text {Dex }}=3\right.$ wt $\%, 0.40 \mathrm{~g}) \mathrm{w} / \mathrm{w} \alpha$-CD solutions were stabilized by PDP as mentioned above. After ultrasonication, before heating and hydrogel formation a droplet of the emulsion $(\sim 0.2 \mathrm{~mL})$ was placed on a microscope slide and Milli-Q water $(\sim 0.2 \mathrm{~mL})$ was added to dilute the emulsion, which breaks the emulsion. However, the dilution by $50 \%$ could not demulsify the emulsion.

Competitive Guest Addition. After hydrogel formation, a competitive guest was added. Anthranilic acid in Milli- $\mathrm{Q}$ water (10 $\mathrm{mM}, 0.5 \mathrm{~mL}$ ) was mixed with compartmentalized hydrogels $(3 \mathrm{~mL})$ and vortexed for $30 \mathrm{~s}$, which led to a sol. Here, there was no demulsification observed under an optical microscope. Finally, the following targeted complete disassembly was performed by diluting $\sim 0.2 \mathrm{~mL}$ of the mixture solution by $100 \%$ with Milli-Q water $(\sim 0.2$ $\mathrm{mL}$ ). When the mixture solution was diluted, a droplet of the solution was directly placed on a microscope slide and complete demulsification was observed (Figure 5d).

Characterization Methods. Freeze drying was applied for hydrogels over $24 \mathrm{~h}$ (LSCbasic, Christ, Germany) to obtain solid samples for observation. Scanning electron microscopy (SEM) (JSM$7500 \mathrm{~F}$ ) was used to visualize the morphology of compartmentalized hydrogels after freeze-drying. The Cryo SEM technique was used to visualize the emulsion droplet with a cryo chamber from Gatan (ALTO 2500). Therefore, the sample was frozen, fractured, and imaged. Fluorescent images were obtained by a confocal laser scanning microscope (CLSM, TCS SP5, Leica, Germany). Prior to visualization, FITC-labeled PEG $\left(<1 \times 10^{-5} \mathrm{~mol} / \mathrm{L}\right)$ was dissolved in the dextran-in-PEG emulsion to fluorescently label the PEG phase. Moreover, the prepared emulsions and compartmentalized hydrogels were imaged by optical microscopy (OM, DM1000 LED, Leica, Germany). The droplet sizes were estimated by ImageJ software. All droplets from one microscopy image were evaluated with the software to obtain a number-average droplet size. Powder X-ray diffraction (XRD) patterns were obtained using Bruker D8 Advance X-ray diffractometer (Billerica, MA) via $\mathrm{Cu} \mathrm{K} \alpha$ radiation. For rheological investigations, compartmentalized hydrogels (or sol after guest addition) were cut into small disc shapes and investigated with an Anton Parr MCR 301 rheometer equipped with a cone plate 12 (CP12) $(d=0.02 \mathrm{~mm})$. Measurements were performed at a constant angular frequency $(10 \mathrm{rad} / \mathrm{s})$ with strain range from 0.1 to $100 \%$ with 

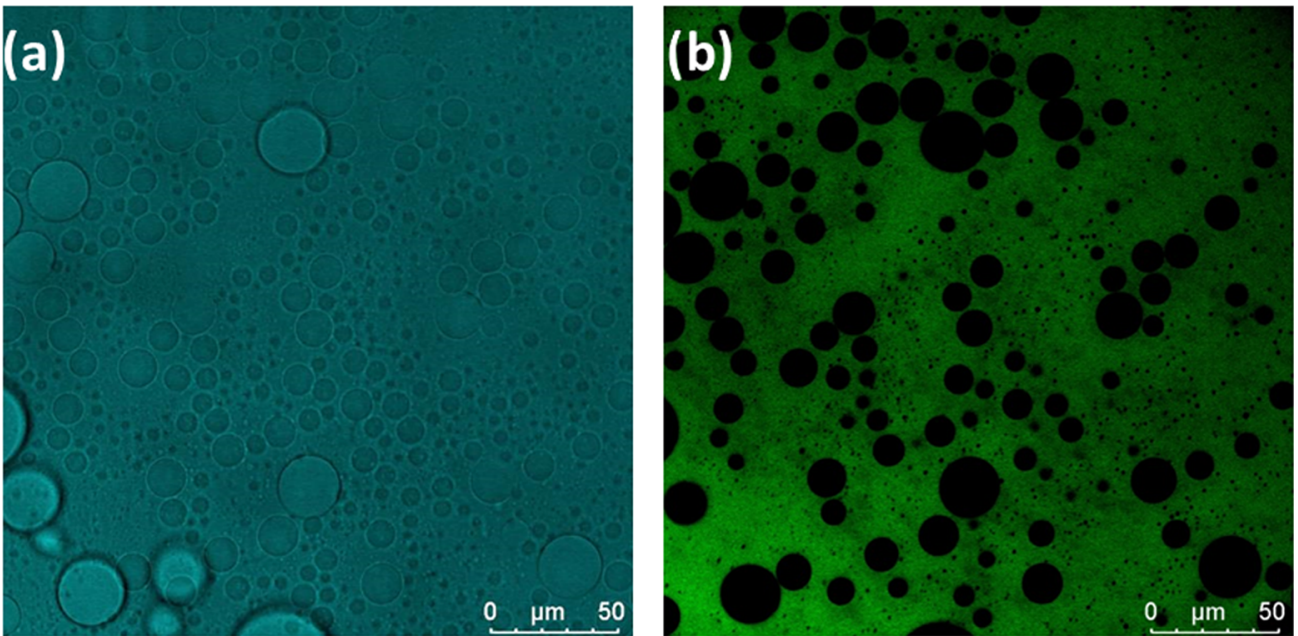

Figure 2. Emulsion droplet images within a compartmentalized hydrogel of the system $\mathrm{PEG}_{35 \mathrm{k}}(7 \mathrm{wt} \%) / \mathrm{dextran}{ }_{40 \mathrm{k}}(3 \mathrm{wt} \%)$; dextran-in-PEG: Bright field images of (a) emulsion droplets within a compartmentalized hydrogel and (b) CLSM image of emulsion droplets within a compartmentalized hydrogel via utilization of FITC-labelled PEG (2k).
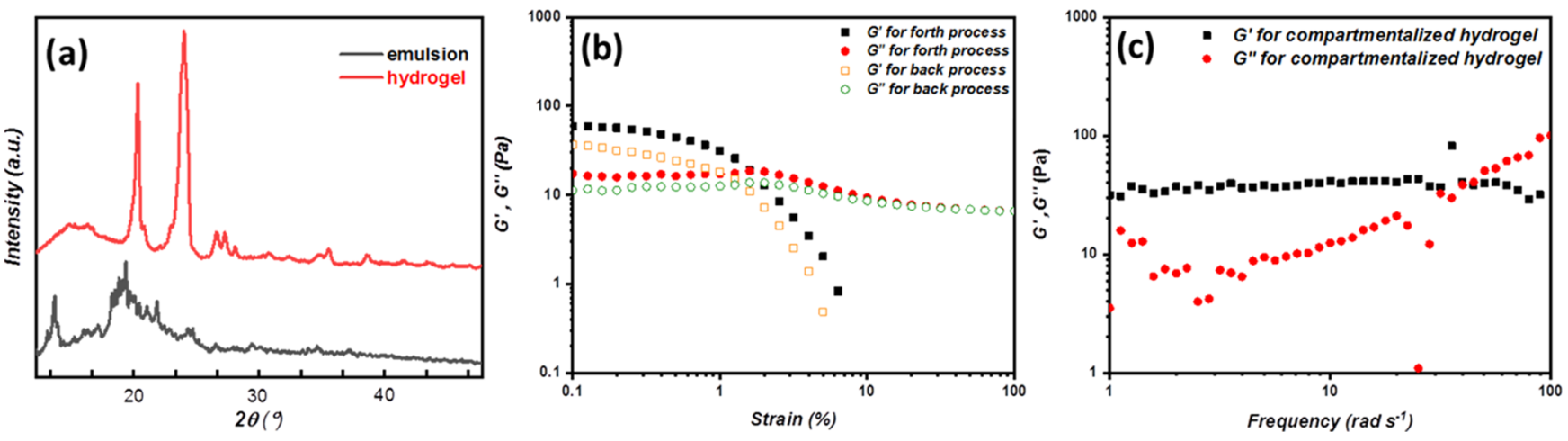

Figure 3. Characterization of compartmentalized hydrogels via PDP $(0.2 \mathrm{~g} / \mathrm{L})$-stabilized $\mathrm{PEG}_{35 \mathrm{k}}(7 \mathrm{wt} \%) /$ dextran $_{40 \mathrm{k}}(3$ wt \%) water-in-water system $(140 \mathrm{mg} / \mathrm{mL}$ of $\alpha$-CD): (a) X-ray diffraction (XRD) patterns of compartmentalized hydrogels and emulsions; (b) strain dependency after heating to $65{ }^{\circ} \mathrm{C}$ and cooling to ambient temperature; and (c) $G^{\prime}$ and $G^{\prime \prime}$ values of hydrogel against frequency with constant strain (0.1\%).

31 measuring points and $0.02 \mathrm{~mm}$ gap. Frequency-dependent measurements were performed at a constant strain $(0.1 \%)$ with changing frequency in the range of $1-100 \mathrm{rad} / \mathrm{s}$. Viscosity measurements were performed at ambient temperature with changing shear rate between 1 and $20 / \mathrm{s}$. The relative error from rheology was estimated to be $2.5 \%$.

\section{RESULTS AND DISCUSSION}

Compartmentalized Hydrogels via PDP-Stabilized Water-in-Water Emulsions. PDPs were prepared by a facile method under alkaline conditions ${ }^{43}$ to produce compartmentalized hydrogels. In a typical experiment, PDPs were fabricated to achieve uniform particles with a size of $400 \mathrm{~nm}$, as previously reported. ${ }^{43}$ Compartmentalized hydrogels were prepared via gelation of $\mathrm{w} / \mathrm{w}$ emulsions stabilized by PDP $(0.2$ $\mathrm{g} / \mathrm{L}$ ) containing various weight ratios of PEG and dextran as well as different molecular weights of PEG and different concentrations of $\alpha$-CD. At first, a PDP suspension was prepared in an $\alpha$-CD solution. Solutions of dextran and PEG were prepared by dissolving the solid in Milli-Q water at neutral $\mathrm{pH}$ with stirring. Finally, the polymer solution was added to the PDP $/ \alpha$-CD mixture (final concentration, $0.2 \mathrm{~g} / \mathrm{L}$ of PDP) for emulsification via ultrasonication and to obtain a hydrogel (Figure 1d). In this way, a compartmentalized hydrogel with well-dispersed compartments could be achieved.
A color difference between emulsions and hydrogels was observed, which was due to slight differences in imaging conditions of optical microscopy as the compartmentalized hydrogel (Figure 1a) is less transparent compared to the emulsion (Figure 1d).

Previously, it has been indicated that PDP-based emulsions show long-term stability for at least 16 weeks. ${ }^{43}$ After ultrasonication, the emulsions were heated to $65{ }^{\circ} \mathrm{C}$ and then cooled to ambient temperature to obtain compartmentalized hydrogels. Investigation via optical microscopy indicated droplet formation inside the hydrogel, which corresponds to a w/w emulsion system, as known from previous studies. However, before cooling to ambient temperature, the mixture was observed under $\mathrm{OM}$ and also frozen for cryo-SEM (Figure $1 \mathrm{~b}, \mathrm{c}$ ) to prove the stability of the emulsion inside the hydrogels as the droplets exist during hydrogel formation. Hence, compartmentalized hydrogels were formed as observed via OM showing w/w droplets in the hydrogels (Figure 1e), which shows that the $\mathrm{w} / \mathrm{w}$ emulsion was stable through the hydrogel formation process, i.e., at a higher temperature. The droplet size inside the hydrogel shows dispersity from 1 to $25 \mu \mathrm{m}$ and the average droplet size is $4 \pm$ $4.0 \mu \mathrm{m}$ (Figure 1e), which matches the observed droplet sizes in corresponding emulsions (the average droplet size $\sim 5 \pm 2.4$ $\mu \mathrm{m}$ in Figure $1 \mathrm{~b}$; it should be noted that the high standard 
deviation is due to the broad dispersity of droplet sizes), showing there is no obvious change during the hydrogel formation even if the temperature varies. To gain further insight on the formed droplets inside the hydrogels and their stability, SEM imaging after freeze-drying of the hydrogel was applied (Figure 1f). Spherical compartments with sizes in the range of $1-25 \mu \mathrm{m}$ were observed, which shows the stability of the compartmentalized architecture even in the dry state.

To further verify the existence of a w/w emulsion stabilized by PDP inside the compartmentalized hydrogels, FITClabelled PEG (2k) was introduced into a dextran-in-PEG emulsion-based hydrogel and imaged via CLSM (Figure 2). The visible dark liquid droplets and clear bright continuous phase region shows the presence of fluorescently labeled PEG continuous phase outside the emulsion droplets, which indicates the separation of PEG and dextran in the hydrogel state, as expected. Confocal microscopy offered a dependable trend for the phase separation contained within the emulsion droplets of a different polymer phase. However, without PDP addition, the droplet structure cannot be observed in the compartmentalized hydrogels (Figure S1), which also confirms the role of PDP as a stabilizer in the $\mathrm{w} / \mathrm{w}$ emulsion. Different characterization methods were applied to show the structure of emulsion droplets and compartmentalized hydrogel; in this way, we can investigate the stability of the compartmentalized hydrogel and give a broader insight into the structure of the fabricated materials.

To gain additional insights into the crosslinking mechanism, XRD measurements of compartmentalized hydrogels were performed after water removal (Figure 3a). ${ }^{44}$ To characterize the crystalline structure of aggregations in the hydrogels, we measured the XRD patterns of a compartmentalized hydrogel in the freeze-dried state and compared them with those from well-dried emulsions without the addition of $\alpha$-CD. In contrast, no signals indicating incorporation of crystalline structures were found for the normal emulsions after freezedrying (Figure 3a). However, the diffraction pattern of the hydrogel exhibits a number of sharp reflections including strong ones at $2 \theta=20.0^{\circ}(d=4.44 \AA)$ and $22.7^{\circ}(d=3.96 \AA)$. These are assigned to the 210 and 300 reflections from the hexagonal lattice with $a=13.6 \AA$. The strong reflection is a typical peak observed for PICs with $\alpha$-CD,,$^{39,45}$ according to the electron density distribution of the core of the $\alpha$-CD molecules with a radius of $\sim 5 \AA$. It is a well-known fact that PEG/ $\alpha$-CD PICs have a channel-type crystalline structure due to the long-chain nature of the guest molecules. These characteristic reflections, which appeared in the profiles from the freeze-dried hydrogels, indicate that the compartmentalized hydrogels are formed via the supramolecular crosslinking. Thus, such crystalline aggregations induced by inclusion complexation formation can be considered to play a major role in the gelation.

Moreover, oscillatory shear rheology was used to investigate the network formation between $\alpha$-CD and PEG. In the case of PDP. The w/w emulsion-based hydrogel $G^{\prime}$ exceeds $G^{\prime \prime}$ in the range of $0.1-100 \%$ of strain, which is a strong indication of hydrogel formation. Albeit the absolute values of $G^{\prime}$ (59.5 Pa at $0.1 \%$ strain) show that rather soft hydrogels are obtained (Figure 3b). The formed hydrogels show significant shearthinning behavior, which is another feature of the supramolecular soft hydrogels. Frequency-dependent rheology measurements in the presence of the hydrogel network did only show a slight change in the region between 0 and $20 \mathrm{rad} / \mathrm{s}$ for both $G^{\prime}$ and $G^{\prime \prime}$, which is due to slow relaxation. However, in the range above $40 \mathrm{rad} / \mathrm{s}$, the loss modulus increased remarkably and finally exceeded $G^{\prime}$ (Figure 3c). Thus, at high frequencies, the supramolecular network turned into a sol. ${ }^{46}$ Besides, viscosity only changed at shear rates between 0 and $10 / \mathrm{s}$, and then no obvious variation was observed for higher shear rates because the gel phase turned into a sol (Figure S2a). However, in the case of emulsions without the addition of $\alpha$-CD, there was no hydrogel formation, which was confirmed by $G^{\prime \prime}$ exceeding $G^{\prime}$ in the range of $0.1-100 \%$ of strain (Figure S2b). Compared with the compartmentalized hydrogels, solely PEG-based hydrogels (Figure S2c) without dextran addition are stable as well. In fact, PEG-based hydrogels were stronger than the compartmentalized hydrogel with dextran. Apparently, the addition of dextran weakened the strength of the hydrogel network but not to an extent to break the network.

Further Studies of Compartmentalized Hydrogel Formation. To investigate the formation of compartmentalized hydrogels further, the parameters of polymer molecular weights were investigated as well as $\alpha$-CD concentration. For the case of PEG with molecular weight less or equal than 3000 $\mathrm{g} / \mathrm{mol}$, no stable hydrogels with $\alpha$-CD were observed (Figure $\mathrm{S} 3 \mathrm{a}){ }^{47}$ which was confirmed by rheology results (Figure S4a) as $G^{\prime \prime}$ exceeds $G^{\prime}$ in the range of strain from 0 to $10 \%$. Nevertheless, precipitate formation at the bottom of the glass vessels was observed. The low molecular weight of PEG is probably not sufficient to support enough $\alpha$-CD for hydrogel formation. Therefore, the formation of hydrogels via inclusion complexes with highly ordered secondary structures should be related to the structural length of PEG in the present system.

In previous works, it was shown that to form an $\alpha$-CD and PEG network, a minimal concentration of $\alpha$-CD of about 50 $\mathrm{mg} / \mathrm{mL}$ is required to obtain a hydrogel with sufficient stiffness to form a self-standing gel. ${ }^{47}$ At lower concentrations (Figure S3b), the emulsion-based $\alpha$-CD network formed with PEG (40k, 7 wt \%) is not strong enough and does not reach a sufficiently high density to resist gravity. The creaming mixture flow at the $\alpha$-CD concentration of $50 \mathrm{mg} / \mathrm{mL}$ indicates that no strong hydrogel network is formed at a concentration below $c_{\alpha-\mathrm{CD}} \leq 50 \mathrm{mg} / \mathrm{mL}$. In the case of the emulsions, the stability of the $\mathrm{w} / \mathrm{w}$ emulsion droplets is enhanced with PDP as stabilizers that attach to the droplets for emulsification. Therefore, creaming was observed instead of sedimentation. On the other hand, when the concentration of $\alpha-\mathrm{CD}$ is increased, raised amounts of $\alpha$-CD/PEG aggregates are formed. The enhanced aggregate accumulation results from an increase in the amount of inclusion complex formation between the PEG chains and $\alpha$-CD molecules. Therefore, the density of the network at steady state increases with raised $\alpha$-CD concentration. If, in addition, this complexation between $\alpha$-CD and PEG is sufficiently strong to resist the buoyancy, creaming no longer takes place. Moreover, it appears from our observations that the network with the embedded emulsion droplets was not sufficiently strong to resist the buoyancy of the droplets for $c_{\alpha-\mathrm{CD}} \geq 200 \mathrm{mg} / \mathrm{mL}$. The collapsed precipitate forms a layer at the bottom of the glass vessels (Figure S3b). Also, no hydrogel formation could be observed in rheology (Figure S5) for $c_{\alpha \text {-CD }}$ $\geq 200 \mathrm{mg} / \mathrm{mL}$. Thus, multicompartment hydrogel formation is observed in the range of $50 \mathrm{mg} / \mathrm{mL} \geq c_{\alpha-\mathrm{CD}} \geq 200 \mathrm{mg} / \mathrm{mL}$, with no network formation at low concentration and demixing at high concentrations. 

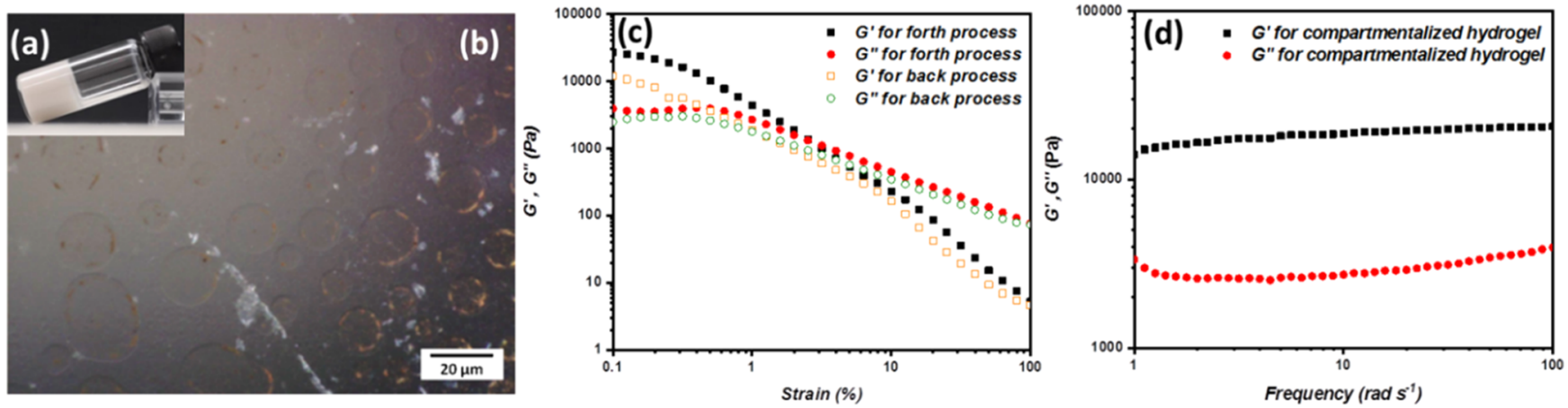

Figure 4. Characterization of the compartmentalized hydrogel via PDP $(0.2 \mathrm{~g} / \mathrm{L})$-stabilized $\mathrm{PEG}_{35 \mathrm{k}}(7 \mathrm{wt} \%) / \mathrm{dextran}$ 40k $(3 \mathrm{wt} \%) \mathrm{w} / \mathrm{w}$ system $(140$ $\mathrm{mg} / \mathrm{mL}$ of $\alpha$-CD) after heating to $90{ }^{\circ} \mathrm{C}$ and cooling to ambient temperature: Optical images of (a) compartmentalized hydrogel; optical microscopy images of (b) emulsion droplets within a compartmentalized hydrogel; (c) strain dependency after heating to $90{ }^{\circ} \mathrm{C}$ and cooling to ambient temperature; and (d) $G^{\prime}$ and $G^{\prime \prime}$ values of hydrogel against frequency with constant strain $(0.1 \%)$.

Furthermore, PEG-dextran aqueous system containing $\alpha$ $\mathrm{CD}(140 \mathrm{mg} / \mathrm{mL})$ and PDP $(0.2 \mathrm{~g} / \mathrm{L})$ was prepared with different ratios of PEG and dextran and hydrogels formed with $\alpha$-CD (Figure S3c). At these compositions, the systems were fully phase-separated with two water phases. ${ }^{43,48}$ Nevertheless, the polymers are not fully separated but enriched in one or the other phase. ${ }^{49}$ Figure S3c shows how the compartmentalized hydrogels in $\alpha$-CD-PDP aqueous system evolved visually with changing PEG/dextran weight ratios. At all weight ratios with dextran in the dispersed phase and PEG in the continuous phase, compartmentalized hydrogels are formed, which is consistent with rheology results (Figure S6). Unexpectedly, hydrogels are also formed in the case of dextran in the continuous phase. Apparently, the residual amount of PEG in the dextran phase is sufficient to form a crosslinked hydrogel although dextran forms the major part of the continuous phase. As shown via a reference experiment, hydrogels can be formed in a dextran phase extracted from PEG/dextran ATPS: Therefore, a PEG/dextran ATPS was formed and the dextran phase-separated (Figure S3d). After addition of $\alpha$-CD, hydrogels were formed, while no hydrogel formation was observed for pure dextran/PDP solutions. After standing overnight, the hydrogels reached a steady state that does not change anymore, indicating that all PEG/dextran ratios in the $\alpha$-CD-PDP aqueous system had produced compartmentalized hydrogels. In addition, there is no obvious effect of the mixture composition on the droplet size (Figure $\mathrm{S} 3 \mathrm{e}-\mathrm{g}$ ). As such, no significant changes in volume fraction and concentration of the two phases were observed $\left(18 \pm 12.6 \mu \mathrm{m}\right.$ for PEG $_{35 \mathrm{k}}$ ( $3 \mathrm{wt}$ $\%) / \operatorname{dextran}_{40 \mathrm{k}}(7 \mathrm{wt} \%), 7 \pm 3.9 \mu \mathrm{m}$ for $\mathrm{PEG}_{35 \mathrm{k}}$ (5 wt $\%) / \operatorname{dextran}_{40 \mathrm{k}}(5 \mathrm{wt} \%)$ and $11 \pm 5.0 \mu \mathrm{m}$ for $\mathrm{PEG}_{35 \mathrm{k}}(7 \mathrm{wt}$ $\%) / \operatorname{dextran}_{40 \mathrm{k}}(3 \mathrm{wt} \%)$; it should be noted that the high standard deviation is due to the broad dispersity of droplet sizes).

Stability of Compartmentalized Hydrogels and Targeted Disassembly. Due to the supramolecular nature of the hydrogel, the application of external stimuli can be utilized to modify the structure. Therefore, a number of methods have been applied here. The hydrogel formation was performed at temperatures around $65{ }^{\circ} \mathrm{C}$. The aqueous dispersion of the polymers, $\alpha$-CD, and PDP became cloudy instantaneously after $2 \mathrm{~h}$ of ultrasonication for emulsification, and the hydrogel formed after heating to $65{ }^{\circ} \mathrm{C}$ and then cooling to ambient temperature. Moreover, these compartmentalized hydrogels featured a phase transition from hydrogel to a clear solution after heating to $65^{\circ} \mathrm{C}$, which is a reversible process as hydrogels formed again at ambient temperature. Another option is to increase the temperature during hydrogel formation, i.e., after ultrasonication, the samples were heated to $90{ }^{\circ} \mathrm{C}$ and then cooled to ambient temperature. Compartmentalized hydrogels could be formed as well, which can be seen from rheology as $G^{\prime}$ exceeds $G^{\prime \prime}$ and strong hydrogels are obtained with respect to high absolute values of $G^{\prime}(27000$ $\mathrm{Pa}$ ) (Figure 4). However, the transition to a solution does not occur again. The compartmentalized hydrogels formed after heat treatment at $90{ }^{\circ} \mathrm{C}$ were heated again but instead of a transparent solution, a turbid sol was observed, which is a suspension containing small presumably crystallite particles. Regarding repeated heating/cooling, rheology was measured after repeated heating to $65{ }^{\circ} \mathrm{C}$ and $G^{\prime}$ reached the same level after cooling. In addition, there was no change for repeated heating and cooling when the hydrogel was prepared after heating to $90{ }^{\circ} \mathrm{C}$. Nevertheless, cooling to ambient temperature resulted in hydrogel formation, which demonstrates that high temperature improves inclusion complex formation as the aggregates do not break completely after heating as observed in the turbid sol character. The increased stability also manifests in the frequency dependency that does not show a crossing of $G^{\prime}$ and $G^{\prime \prime}$ at increased frequencies as it was evident for the hydrogel prepared after heating to $65{ }^{\circ} \mathrm{C}$. However, the compartmentalized hydrogels could not be formed at heating temperatures below $65{ }^{\circ} \mathrm{C}$ (Figure S7a). In addition, no significant impact of heating time on the hydrogel formation was found. Emulsions kept at an elevated temperature $\left(65^{\circ} \mathrm{C}\right)$ for different times, 1, 5, 10 and $30 \mathrm{~min}$ (Figure S7b), turned all into hydrogels.

Disassembly of the compartmentalized hydrogels can be also obtained by applying the addition of competitive guests (Figure 5a). Therefore, $10 \mathrm{mM}$ anthranilic acid was introduced into the supramolecular compartmentalized hydrogels and the mixture was vortexed for $30 \mathrm{~s}$. The obtained flowing turbid liquid confirms the successful destruction of the hydrogel network structure. The introduction of competitive guest, i.e., anthranilic acid, has a profound competitive effect on the $\alpha$ $\mathrm{CD} / \mathrm{PEG}$ PICs. As the addition of additional guests interacts with the present $\alpha$-CD capacities the equilibrium is shifted, which leads to inclusion complexations between $\alpha-\mathrm{CD}$ and anthranilic acid. Thus, the hydrogel disassembly of the hydrogel is initiated as the PEG is expelled from the $\alpha$-CD cavities. Hence, it is indicated that disassembly of the hydrogel structure via competitive guest addition is possible. Most importantly, the aqueous two-phase emulsions are still stable 


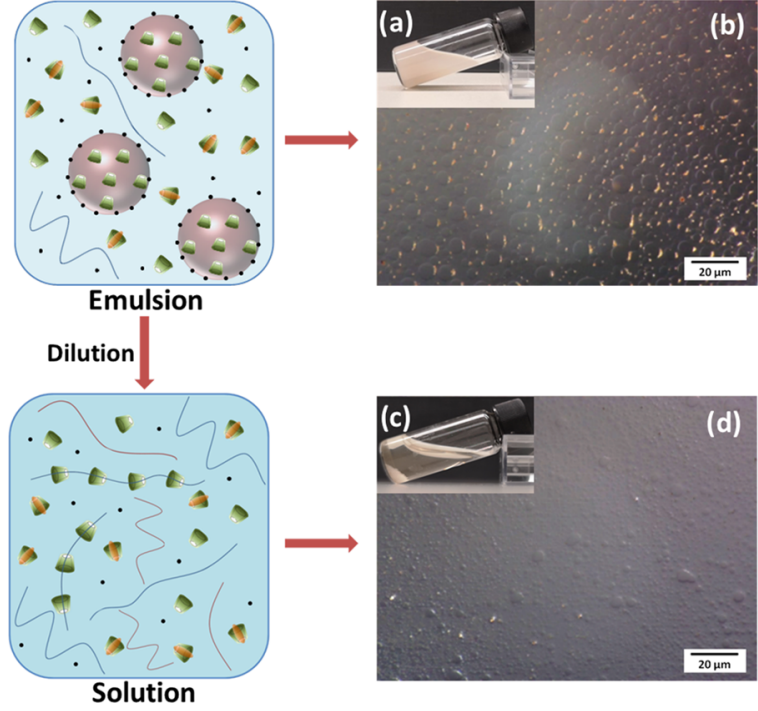

Figure 5. Characterization of the compartmentalized hydrogel via PDP $(0.2 \mathrm{~g} / \mathrm{L})$ stabilized $\mathrm{PEG}_{35 \mathrm{k}}(7 \mathrm{wt} \%) / \operatorname{dextran}_{40 \mathrm{k}}(3 \mathrm{wt} \%)$ waterin-water system $\left(140 \mathrm{mg} / \mathrm{mL}\right.$ of $\alpha$-CD) after heating to $90{ }^{\circ} \mathrm{C}$ and cooling to the ambient temperature: Optical images of (a) compartmentalized hydrogel after adding the competitive guest and (c) the following dilution by $100 \%$; optical microscopy images of (b) droplets within an emulsion after adding the competitive guest and (d) the following dilution by $100 \%$.

after hydrogel disassembly (Figure $5 b$ ). In order to quantify the required amount of anthranilic acid to break the hydrogels, the concentrations of anthranilic acid were screened between 4 and $12 \mathrm{mM}$ (Figure S8). It could be shown that a minimum of $10 \mathrm{mM}$ anthranilic acid is needed to disassemble the hydrogels. However, after addition of competitive guest and dilution by $100 \%$, the emulsion breaks as no droplets can be observed via optical microscopy anymore (Figure $5 \mathrm{c}, \mathrm{d}$ ), which is expected due to the shift out of the two-phase region in the phase diagram. ${ }^{43}$ Moreover, the oscillatory shear rheology indicates hydrogel cleavage as $G^{\prime \prime}$ exceeds $G^{\prime}$ (Figure S9). The confocal images showed disassembly and demulsification after guest addition and dilution as well (Figure S10). No complete droplets could be observed via confocal microscope anymore. However, ill-defined aggregates were visible, which might be corresponding to various $\alpha$-CD complexes. Therefore, the state of the system can be tailored from compartmentalized hydrogel to emulsion and further to solution.

\section{CONCLUSIONS}

A facile, generic approach is presented for the fabrication of compartmentalized completely hydrophilic materials by forming a supramolecular hydrogel within mixtures of aqueous phase-separating polymers, in the presence of Pickering-type PDP stabilizers, dextran, PEG, and $\alpha$-CD. PEG is found to form inclusion complexes with $\alpha$-CD molecules in the continuous phase, resulting in hydrogel formation, and in physical compartments from emulsion droplets. The compartmentalized hydrogels were assessed via SEM, OM, and CLSM for morphology. The origin of hydrogel formation was confirmed via XRD and the gelation followed by oscillatory shear rheology. By varying the different constituent parts of the hydrogels, e.g., the molecular mass of the utilized polymers, the polymer concentration, and $\alpha$-CD concentration as well as the temperature, the properties of the structure could be varied.
After the addition of a competitive guest, the original network can be disassembled. Notably, after disassembly of the hydrogel via competitive guests, the emulsion stays intact and the emulsion finally breaks after significant dilution. As such, the system can be tuned in a multilevel way from hydrogel to emulsion to solution via external manipulations. Overall, this method can be applied to several all-aqueous emulsions. Such types of compartmentalized hydrogels could potentially provide new opportunities for a wide variety of aqueous multiphase systems, in the design of novel biomimetic hydrogel catalysts, as the templates of porous soft materials or in the fabrication of supramolecular hydrogel scaffolds for tissue engineering.

\section{ASSOCIATED CONTENT}

\section{Supporting Information}

The Supporting Information is available free of charge on the ACS Publications website at DOI: 10.1021/acs.langmuir.9b01101.

Synthetic procedures, rheology, and photographs (PDF)

\section{AUTHOR INFORMATION}

\section{Corresponding Author}

*E-mail: bernhard.schmidt@mpikg.mpg.de, bernhard. schmidt@glasgow.ac.uk. Tel: (+44) 1413308469.

\section{ORCID}

Bernhard V. K. J. Schmidt: 0000-0002-3580-7053

\section{Author Contributions}

The manuscript was written through contributions of all authors. All authors have given approval to the final version of the manuscript.

\section{Notes}

The authors declare no competing financial interest.

\section{ACKNOWLEDGMENTS}

The authors are grateful for funding from the Max-Planck society. J.Z. acknowledges financial support from the China Scholarship Council. The authors acknowledge Heike Runge for assistance with cryogenic SEM measurements and Carmen Remde for assistance with LSCM measurements.

\section{REFERENCES}

(1) Leamon, J. H.; Link, D. R.; Egholm, M.; Rothberg, J. M. Overview: methods and applications for droplet compartmentalization of biology. Nat. Methods 2006, 3, 541.

(2) Chaffey, N.; Alberts, B.; Johnson, A.; Lewis, J.; Raff, M.; Roberts, K.; Walter, P. Molecular biology of the cell. 4th edn. Ann. Bot. 2003, 91, 401.

(3) Marguet, M.; Bonduelle, C.; Lecommandoux, S. Multicompartmentalized polymeric systems: towards biomimetic cellular structure and function. Chem. Soc. Rev. 2013, 42, 512-529.

(4) Gaitzsch, J.; Huang, X.; Voit, B. Engineering Functional Polymer Capsules toward Smart Nanoreactors. Chem. Rev. 2016, 116, 10531093.

(5) Chen, A. H.; Silver, P. A. Designing biological compartmentalization. Trends Cell Biol. 2012, 22, 662-670.

(6) Schoonen, L.; van Hest, J. C. M. Compartmentalization Approaches in Soft Matter Science: From Nanoreactor Development to Organelle Mimics. Adv. Mater. 2016, 28, 1109-1128.

(7) Li, M.; Huang, X.; Tang, T. D.; Mann, S. Synthetic cellularity based on non-lipid micro-compartments and protocell models. Curr. Opin. Chem. Biol. 2014, 22, 1-11. 
(8) Kelly, B. T.; Baret, J.-C.; Taly, V.; Griffiths, A. D. Miniaturizing chemistry and biology in microdroplets. Chem. Commun. 2007, 18, 1773-1788.

(9) Massignani, M.; Lomas, H.; Battaglia, G. Polymersomes: A Synthetic Biological Approach to Encapsulation and Delivery. In Modern Techniques for Nano- and Microreactors/-reactions; Caruso, F., Ed.; Springer: Berlin, 2010; pp 115-154.

(10) Heinz, D.; Amado, E.; Kressler, J. Polyphilicity-An Extension of the Concept of Amphiphilicity in Polymers. Polymers 2018, 10, 960.

(11) Li, Z.; Kesselman, E.; Talmon, Y.; Hillmyer, M. A.; Lodge, T. P. Multicompartment Micelles from ABC Miktoarm Stars in Water. Science 2004, 306, 98-101.

(12) Fernandez-Trillo, F.; Grover, L. M.; Stephenson-Brown, A.; Harrison, P.; Mendes, P. M. Vesicles in Nature and the Laboratory: Elucidation of Their Biological Properties and Synthesis of Increasingly Complex Synthetic Vesicles. Angew. Chem., Int. Ed. 2017, 56, 3142-3160.

(13) Keating, C. D. Aqueous Phase Separation as a Possible Route to Compartmentalization of Biological Molecules. Acc. Chem. Res. 2012, $45,2114-2124$

(14) Schmidt, B. V. K. J. Double Hydrophilic Block Copolymer SelfAssembly in Aqueous Solution. Macromol. Chem. Phys. 2018, 219, No. 1700494

(15) Ding, P.; Wolf, B.; Frith, W.; Clark, A.; Norton, I.; Pacek, A. W. Interfacial tension in phase-separated gelatin/dextran aqueous mixtures. J. Colloid Interface Sci. 2002, 253, 367-376.

(16) Grilo, A. L.; Raquel Aires-Barros, M.; Azevedo, A. M. Partitioning in Aqueous Two-Phase Systems: Fundamentals, Applications and Trends. Sep. Purif. Rev. 2016, 45, 68-80.

(17) Walter, H.; Johansson, G. Partitioning in aqueous two-phase systems: an overview. Anal. Biochem. 1986, 155, 215-242.

(18) Aumiller, W. M.; Keating, C. D. Experimental models for dynamic compartmentalization of biomolecules in liquid organelles: Reversible formation and partitioning in aqueous biphasic systems. Adv. Colloid Interface Sci. 2017, 239, 75-87.

(19) Aumiller, W. M.; Davis, B. W.; Hashemian, N.; Maranas, C.; Armaou, A.; Keating, C. D. Coupled Enzyme Reactions Performed in Heterogeneous Reaction Media: Experiments and Modeling for Glucose Oxidase and Horseradish Peroxidase in a PEG/Citrate Aqueous Two-Phase System. J. Phys. Chem. B 2014, 118, 2506-2517.

(20) Cacace, D. N.; Rowland, A. T.; Stapleton, J. J.; Dewey, D. C.; Keating, C. D. Aqueous Emulsion Droplets Stabilized by Lipid Vesicles as Microcompartments for Biomimetic Mineralization. Langmuir 2015, 31, 11329-11338.

(21) Ganley, W. J.; Ryan, P. T.; van Duijneveldt, J. S. Stabilisation of water-in-water emulsions by montmorillonite platelets. J. Colloid Interface Sci. 2017, 505, 139-147.

(22) Peddireddy, K. R.; Nicolai, T.; Benyahia, L.; Capron, I. Stabilization of water-in-water emulsions by nanorods. ACS Macro Lett. 2016, 5, 283-286.

(23) Vis, M.; Opdam, J.; van 't Oor, I. S. J.; Soligno, G.; van Roij, R.; Tromp, R. H.; Erné, B. H. Water-in-Water Emulsions Stabilized by Nanoplates. ACS Macro Lett. 2015, 4, 965-968.

(24) Bai, S.; Pappas, C.; Debnath, S.; Frederix, P. W. J. M.; Leckie, J.; Fleming, S.; Ulijn, R. V. Stable Emulsions Formed by Self-Assembly of Interfacial Networks of Dipeptide Derivatives. ACS Nano 2014, 8, 7005-7013.

(25) Moreira, I. P.; Sasselli, I. R.; Cannon, D. A.; Hughes, M.; Lamprou, D. A.; Tuttle, T.; Ulijn, R. V. Enzymatically activated emulsions stabilised by interfacial nanofibre networks. Soft Matter 2016, 12, 2623-2631.

(26) Saini, P. Multi-phase systems: Hydrogel-driven compartmentalization. Nat. Rev. Chem. 2017, 1, No. 0084.

(27) Mytnyk, S.; Olive, A. G. L.; Versluis, F.; Poolman, J. M.; Mendes, E.; Eelkema, R.; van Esch, J. H. Compartmentalizing Supramolecular Hydrogels Using Aqueous Multi-phase Systems. Angew. Chem., Int. Ed. 2017, 129, 15119-15123.
(28) Ben Ayed, E.; Cochereau, R.; Dechancé, C.; Capron, I.; Nicolai, T.; Benyahia, L. Water-In-Water Emulsion Gels Stabilized by Cellulose Nanocrystals. Langmuir 2018, 34, 6887-6893.

(29) Gonzalez-Jordan, A.; Benyahia, L.; Nicolai, T. Cold gelation of water in water emulsions stabilized by protein particles. Colloids Surf., A 2017, 532, 332-341.

(30) Shum, H. C.; Weitz, D. A. Multicompartment polymersome gel for encapsulation. Soft Matter 2011, 7, 8762-8765.

(31) Wang, C.; Zhang, G.; Liu, G.; Hu, J.; Liu, S. Photo- and thermo-responsive multicompartment hydrogels for synergistic delivery of gemcitabine and doxorubicin. J. Controlled Release 2017, 259, 149-159.

(32) Harada, A. Preparation and structures of supramolecules between cyclodextrins and polymers. Coord. Chem. Rev. 1996, 148, 115-133.

(33) Appel, E. A.; del Barrio, J.; Loh, X. J.; Scherman, O. A. Supramolecular polymeric hydrogels. Chem. Soc. Rev. 2012, 41, 61956214.

(34) Murakami, T.; Schmidt, B. V. K. J.; Brown, H. R.; Hawker, C. J. One-Pot "Click" Fabrication of Slide-Ring Gels. Macromolecules 2015, 48, 7774-7781.

(35) Liu, G.; Yuan, Q.; Hollett, G.; Zhao, W.; Kang, Y.; Wu, J. Cyclodextrin-based host-guest supramolecular hydrogel and its application in biomedical fields. Polym. Chem. 2018, 9, 3436-3449.

(36) Schmidt, B. V. K. J.; Barner-Kowollik, C. Dynamic Macromolecular Material Design-The Versatility of Cyclodextrin-Based Host-Guest Chemistry. Angew. Chem., Int. Ed. 2017, 56, 8350-8369.

(37) Hashidzume, A.; Harada, A. Recognition of polymer side chains by cyclodextrins. Polym. Chem. 2011, 2, 2146-2154.

(38) Huh, K. M.; Ooya, T.; Lee, W. K.; Sasaki, S.; Kwon, I. C.; Jeong, S. Y.; Yui, N. Supramolecular-Structured Hydrogels Showing a Reversible Phase Transition by Inclusion Complexation between Poly(ethylene glycol) Grafted Dextran and $\alpha$-Cyclodextrin. Macromolecules 2001, 34, 8657-8662.

(39) Li, T.; Kumru, B.; Al Nakeeb, N.; Willersinn, J.; Schmidt, B. V. K. J. Thermoadaptive Supramolecular $\alpha$-Cyclodextrin CrystallizationBased Hydrogels via Double Hydrophilic Block Copolymer Templating. Polymers 2018, 10, 576.

(40) Bleta, R.; Menuel, S.; Léger, B.; Da Costa, A.; Monflier, E.; Ponchel, A. Evidence for the existence of crosslinked crystalline domains within cyclodextrin-based supramolecular hydrogels through sol-gel replication. RSC Adv. 2014, 4, 8200-8208.

(41) Cui, J.; Yan, Y.; Such, G. K.; Liang, K.; Ochs, C. J.; Postma, A.; Caruso, F. Immobilization and Intracellular Delivery of an Anticancer Drug Using Mussel-Inspired Polydopamine Capsules. Biomacromolecules 2012, 13, 2225-2228.

(42) Lee, H.; Dellatore, S. M.; Miller, W. M.; Messersmith, P. B. Mussel-inspired surface chemistry for multifunctional coatings. Science 2007, 318, 426-430.

(43) Zhang, J.; Hwang, J.; Antonietti, M.; Schmidt, B. V. K. J. Waterin-Water Pickering Emulsion Stabilized by Polydopamine Particles and Cross-Linking. Biomacromolecules 2019, 20, 204-211.

(44) Al Nakeeb, N.; Kochovski, Z.; Li, T.; Zhang, Y.; Lu, Y.; Schmidt, B. V. K. J. Poly(ethylene glycol) brush-b-poly(N-vinylpyrrolidone)-based double hydrophilic block copolymer particles crosslinked via crystalline $\alpha$-cyclodextrin domains. RSC Adv. 2019, 9, 4993-5001.

(45) Huh, K. M.; Ooya, T.; Lee, W. K.; Sasaki, S.; Kwon, I. C.; Jeong, S. Y.; Yui, N. Supramolecular-structured hydrogels showing a reversible phase transition by inclusion complexation between poly (ethylene glycol) grafted dextran and $\alpha$-cyclodextrin. Macromolecules 2001, 34, 8657-8662.

(46) Iohara, D.; Okubo, M.; Anraku, M.; Uramatsu, S.; Shimamoto, T.; Uekama, K.; Hirayama, F. Hydrophobically Modified Polymer $/ \alpha$ Cyclodextrin Thermoresponsive Hydrogels for Use in Ocular Drug Delivery. Mol. Pharmaceutics 2017, 14, 2740-2748.

(47) Harada, A.; Li, J.; Kamachi, M. Preparation and properties of inclusion complexes of polyethylene glycol with .alpha.-cyclodextrin. Macromolecules 1993, 26, 5698-5703. 
(48) Mace, C. R.; Akbulut, O.; Kumar, A. A.; Shapiro, N. D.; Derda, R.; Patton, M. R.; Whitesides, G. M. Aqueous Multiphase Systems of Polymers and Surfactants Provide Self-Assembling Step-Gradients in Density. J. Am. Chem. Soc. 2012, 134, 9094-9097.

(49) Dimova, R.; Lipowsky, R. Lipid membranes in contact with aqueous phases of polymer solutions. Soft Matter 2012, 8, 64096415. 